\title{
Role of genes involved in lignin biosynthesis in flax response to Fusarium oxysporum
}

\author{
R.O. Novakovskiy ${ }^{1 *}$, G.S. Krasnov ${ }^{1}$, T.A. Rozhmina ${ }^{1,2}$, L.P. Kudryavtseva ${ }^{2}$, A.A. Zhuchenko ${ }^{2,3}$, E.N. Pushkova ${ }^{1}$, L.V. Povkhova ${ }^{1,4}$, \\ P. Kezimana ${ }^{1,5}$, A.A. Dmitriev ${ }^{1}$, N.V. Melnikova ${ }^{1}$
}

\author{
${ }^{1}$ Engelhardt Institute of Molecular Biology, Russian Academy of Sciences, Moscow, Russia \\ ${ }^{2}$ Federal Research Center for Bast Fiber Crops, Torzhok, Russia \\ ${ }^{3}$ All-Russian Horticultural Institute for Breeding, Agrotechnology and Nursery, Moscow, Russia \\ ${ }^{4}$ Moscow Institute of Physics and Technology, Dolgoprudny, Russia \\ ${ }^{5}$ Peoples' Friendship University of Russia (RUDN University), Moscow, Russia
}

DOI 10.18699/ICG-PlantGen2019-26

(c) Autors, 2019

* e-mail: Olegovich46@mail.ru

\begin{abstract}
Flax (Linum usitatissimum L.) is an important crop used in various industries. Fusarium oxysporum is the causative agent of Fusarium wilt, and this disease is the most harmful for flax. The most effective way to control this pathogen is to develop resistant varieties. The mechanisms of flax resistance to $F$. oxysporum are still unclear. In our work, we analyzed the data obtained by transcriptome sequencing of resistant and susceptible flax varieties grown under control conditions and after inoculation with $F$. oxysporum. We evaluated expression alterations of $C C R, C C O A O M T, C O M T$, and $4 C L$ gene families, which are involved in lignin biosynthesis, and revealed their significant upregulation in flax varieties in response to F. oxysporum.
\end{abstract}

Key words: flax; Linum usitatissimum; resistant cultivars; Fusarium oxysporum; RNA-Seq; transcriptome; CCR; CCOAOMT; COMT; $4 \mathrm{CL}$.

\section{Introduction}

Flax (Linum usitatissimum L.) is a crop that is a source of fiber and flaxseed (Singh et al., 2011; Goyal et al., 2014). Fusarium oxysporum f. sp. lini is the most harmful pathogen that decreases the quality of flax products (Rashid, 2003). The molecular mechanisms of the resistance of L. usitatissimum to $F$. oxysporum are still unclear, and the search for genes involved in the response of flax to the pathogen is the main purpose of the study. The involvement of proteins associated with pathogenesis in the flax response to $F$. oxysporum infection was revealed (Wrobel-Kwiatkowska et al., 2004; Wojtasik et al., 2014; Galindo-Gonzalez, Deyholos, 2016).

In response to the infection with the fungus Fusarium oxysporum, cell walls are lignified. Many genes are involved in the process of lignin biosynthesis, including cinnamoyl CoA reductase $(C C R)$, caffeoyl-CoA O-methyltransferase (CCoAOMT), catechol-O-methyltransferase (COMT), 4-coumaric acid: coenzyme A ligase $(4 C L)$. Each of these genes has homologs located on different chromosomes (Wojtasik et al., 2015; Boba et al., 2016; Wojtasik et al., 2016).

Plants with reduced regulation of these genes were less likely to tolerate the disease caused by F. oxysporum (Hano et al., 2006). In the present work, we evaluated expression alterations of $C C R, C C O A O M T, C O M T$, and $4 C L$ gene families under $F$. oxysporum infection using RNA-Seq data for resistant and susceptible flax varieties. This made it possible to determine the general trends in the response of Linum usitatissimum to the pathogen and genotype-specific changes in expression.

\section{Materials and methods}

As the experimental material, resistant (3896 and 'Dakota') and susceptible (TOST and AP5) flax varieties were used, as well as their stable $\mathrm{BC}_{2} \mathrm{~F}_{5}$ hybrids $(3896 \times \mathrm{AP} 5$, recurrent parent AP5, and 'Dakota' $\times$ AP5, recurrent parent AP5). Seed material was provided by the Institute for Flax (Torzhok, Russia). For seed sterilization, rinsing with $70 \%$ ethanol for 1 minute and $1 \%$ sodium hypochlorite for 20 minutes were used. For growing flax plants from seeds, Murashige-Skoog medium poured into $15-\mathrm{ml}$ glass tubes was used.

The tubes with the medium and seeds were incubated at $22{ }^{\circ} \mathrm{C}$ with a photoperiod of 16-hour day/8-hour night for seven days. After a week, half of the samples were infected with the most pathogenic isolate, $F$. oxysporum isolate \#39, from the phytopathogen collection of the Institute for Flax. The second half of the non-infected plants acted as the control. After 48 hours, 240 plants were removed from the tubes and the tips of the roots were collected followed by freezing in liquid nitrogen. RNA was then extracted from plant pools of 10-12 plants using the RNeasy Plant Mini Kit (Qiagen, USA).

As a result, we obtained 24 samples of RNA: TOST, AP5, 3896, 'Dakota', $3896 \times$ AP5, 'Dakota' $\times$ AP5 under control and experimental conditions in duplicate. Using an Agilent 2100 bioanalyzer (Agilent Technologies, USA) and a Qubit 2.0 fluorometer (Life Technologies, USA), we evaluated the quality and quantity of the RNA obtained. To obtain a cDNA library, a TruSeq Stranded Total RNA Sample Prep Kit (Illumina, USA) was used. Sequencing was performed on NextSeq500 (Illumina) with 80-nucleotide paired-end reads. The Trimmomatic program (Bolger et al., 2014) was used for trimming reads. Then, filtration was carried out with the Fusarium oxysporum reference genome and the rest reads were mapped to the L. usitatissimum reference genome (GenBank assembly GCA_000224295.2) using STAR (Dobin et al., 2013) and quantified using BEDTools (Quinlan and Hall, 2010).

The CPM values were determined for each gene for each variety under control and infection conditions. After that, the $\log$ (CPM Fusarium / CPM control) value was calculated for each variety and the $\mathrm{BC}_{2} \mathrm{~F}_{5}$ population. This work was done 
Table 1

Expression alterations of $C C R, C C O A O M T, C O M T, 4 C L$ genes in flax cultivars and populations with diverse resistance to $F$. oxysporum

\begin{tabular}{|c|c|c|c|c|c|c|}
\hline \multirow{3}{*}{ Gene } & \multicolumn{6}{|c|}{$\log (C P M$ Fusarium/CPM control) } \\
\hline & \multicolumn{2}{|c|}{ Susceptible genotypes } & \multicolumn{4}{|c|}{ Resistant genotypes } \\
\hline & TOST & AP5 & 3896 & Dakota & $3896 \times$ AP5 & Dakota $\times$ AP5 \\
\hline$C C R(\mathrm{Lu} 4)$ & 0.12 & 0.17 & 0.31 & 0.36 & 0.09 & 0.36 \\
\hline CCR (Lu5) & 0.47 & 0.45 & 1.01 & 0.60 & 0.50 & 0.70 \\
\hline CCoAOMT (Lu6) & 0.50 & 0.53 & 1.27 & 0.40 & 0.46 & 0.77 \\
\hline CCOAOMT (Lu8) & 1.03 & 1.28 & 1.74 & 1.42 & 0.90 & 1.39 \\
\hline COMT (Lu1) & 1.41 & 1.41 & 1.81 & 1.36 & 0.69 & 1.35 \\
\hline COMT(Lu14) & 1.17 & 1.36 & 1.44 & 1.18 & 0.61 & 1.42 \\
\hline $4 C L(\mathrm{Lu} 2)$ & 0.28 & 1.04 & 0.78 & 1.11 & 0.38 & 0.82 \\
\hline $4 C L(\mathrm{Lu} 4)$ & 0.06 & -0.17 & 0.20 & 0.21 & -0.06 & -0.28 \\
\hline $4 C L$ (Lu5) & 0.09 & 0.23 & 0.92 & 0.19 & 0.32 & 0.63 \\
\hline $4 C L(\operatorname{Lu} 13)$ & 0.99 & 0.92 & 1.38 & 1.24 & 0.49 & 1.21 \\
\hline
\end{tabular}

using the equipment of the Genome Center of the Engelhardt Institute of Molecular Biology (http://www.eimb.ru/rus/ckp/ ccu_genome_c.php).

\section{Results and discussion}

To identify genes in the CCR, CCOAOMT, COMT, and $4 C L$ families, we took the sequences of these genes and used NCBI BLAST to find homologous sequences in the reference Linum usitatissimum genome. As a result, we identified the numbers of homologs of the genes on different chromosomes: 2 for $C C R, 2$ for CCOAOMT, 2 for COMT, and 4 for $4 C L$. To denote the chromosome number, we used the designation "(Lu\#)", where "\#" is the number of the chromosome according to the reference assembly.

For each gene, the expression was evaluated on the basis of RNA-Seq data obtained for six varieties and populations of flax that were grown under control conditions or with $\mathrm{Fu}$ sarium oxysporum isolate \#39 (Dmitriev et al., 2017). Results are shown in Table 1, where log (CPM Fusarium/CPM control) values for each gene studied are presented for resistant (3896, 'Dakota', $3896 \times$ AP5, 'Dakota' × AP5) and susceptible (TOST and AP5) genotypes under control conditions and 48 hours after inoculation with Fusarium oxysporum.

A statistically significant increase in expression in flax varieties under $F$. oxysporum infection compared to the control conditions was observed for all studied genes except $4 C L$ (Lu4) ( $p<0.05$, Mann-Whitney test). The most significant increase in expression was detected for CCOAOMT (Lu8), COMT (Lu1), COMT (Lu14), and 4CL (Lu13) genes - more than 2-fold upregulation on average. There were no statistically significant differences in expression alterations between resistant and susceptible genotypes.

\section{Conclusions}

The search for genes with diverse expression changes in resistant and susceptible genotypes under Fusarium oxysporum infection is important for identifying resistance genes. In the present work, we used our RNA-Seq data for flax genotypes resistant (3896, 'Dakota', $3896 \times$ AP5, 'Dakota' $\times$ AP5) and susceptible (TOST and AP5) to F. oxysporum in control conditions or 48 hours after inoculation with $F$. oxysporum to evaluate the expression of 10 homologs of the CCR, CCOAOMT, $C O M T$, and $4 C L$ genes involved in lignin biosynthesis. We revealed that 9 of these genes were characterized by upregulation upon infection with the pathogen in all the genotypes studied. However, no genotype-specific changes were observed. The results of this work indicate the involvement of the $C C R$, $C C O A O M T, C O M T$, and $4 C L$ gene families in the response to Fusarium oxysporum infection. Our data also contribute to the understanding of the role of the CCR, CCOAOMT, COMT, and $4 C L$ genes in the stress response and resistance.

\section{References}

Boba A., Kostyn K., Kostyn A. et al. Methyl Salicylate Level Increase in Flax after Fusarium oxysporum Infection Is Associated with Phenylpropanoid Pathway Activation. Front Plant Sci. 2016;7:1951. DOI 10.3389/fpls.2016.01951.

Bolger A.M., Lohse M., Usadel B. Trimmomatic: a flexible trimmer for Illumina sequence data. Bioinformatics. 2014;30(15):2114-2120. DOI 10.1093/bioinformatics/btu170.

Dmitriev A.A., Krasnov G.S., Rozhmina T.A. et al. Differential gene expression in response to Fusarium oxysporum infection in resistant and susceptible genotypes of flax (Linum usitatissimum L.). BMC Plant Biol. 2017;17(Suppl 2):253. DOI 10.1186/s12870-017-1192-2.

Dobin A., Davis C.A., Schlesinger F. et al. STAR: ultrafast universal RNA-seq aligner. Bioinformatics. 2013;29(1):15-21. DOI 10.1093/ bioinformatics/bts635.

Galindo-Gonzalez L., Deyholos M.K. RNA-seq Transcriptome Response of Flax (Linum usitatissimum L.) to the Pathogenic Fungus Fusarium oxysporum f. sp. lini. Front Plant Sci. 2016;7:1766. DOI $10.3389 /$ fpls.2016.01766.

Goyal A., Sharma V., Upadhyay N. et al. Flax and flaxseed oil: an ancient medicine \& modern functional food. J Food Sci Technol. 2014;51(9):1633-1653. DOI 10.1007/s13197-013-1247-9.

Hano C., Addi M., Bensaddek L. et al. Differential accumulation of monolignol-derived compounds in elicited flax (Linum usitatissi- 
mum) cell suspension cultures. Planta. 2006;223(5):975-989. DOI 10.1007/s00425-005-0156-1.

Quinlan A.R., Hall I.M. BEDTools: a flexible suite of utilities for comparing genomic features. Bioinformatics. 2010;26(6):841-842. DOI 10.1093/bioinformatics/btq033.

Rashid K. Principal diseases of flax. Flax, CRC Press: 2003;92-123.

Singh K.K., Mridula D., Rehal J. et al. Flaxseed: a potential source of food, feed and fiber. Crit Rev Food Sci Nutr. 2011;51(3):210-222. DOI 10.1080/10408390903537241.

Wojtasik W., Kulma A., Boba A. et al. Oligonucleotide treatment causes flax beta-glucanase up-regulation via changes in gene-body methylation. BMC Plant Biol. 2014;14:261. DOI 10.1186/s12870-0140261-z.

Wojtasik W., Kulma A., Dyminska L. et al. Evaluation of the significance of cell wall polymers in flax infected with a pathogenic strain of Fusarium oxysporum. BMC Plant Biol. 2016;16:75. DOI 10.1186/s12870-016-0762-z.

Wojtasik W., Kulma A., Namysl K., et al. Polyamine metabolism in flax in response to treatment with pathogenic and non-pathogenic Fusarium strains. Front Plant Sci. 2015;6:291. DOI 10.3389/fpls. 2015.00291 .

Wrobel-Kwiatkowska M., Lorenc-Kukula K., Starzycki M. et al. Expression of beta-1,3-glucanase in flax causes increased resistance to fungi. Physiological Mol. Plant Pathol. 2004;65(5):245-256. DOI 10.1016/j.pmpp.2005.02.008.

Acknowledgements. This work was financially supported by the Russian Science Foundation, grant 16-16-00114.

Conflict of interest. The authors declare no conflict of interest. 